\title{
Research and Development of Superhard Cutting Database
}

\author{
HuiPing Zhang ${ }^{1 \mathrm{a}}$, WenJuan Zheng ${ }^{1 \mathrm{~b}}$, YiNan Lai ${ }^{1 \mathrm{c}}$, Mingna Ding ${ }^{1 \mathrm{~d}}$ and Zhen Chen ${ }^{1 \mathrm{e}}$ \\ ${ }^{1}$ College of Mechanical and Power Engineering,Harbin University of Science and \\ Technology,Harbin 150080, China \\ azhping302@163.com; ${ }^{b}$ zhengwenjuanjuan@163.com; ${ }^{c}$ laiyinan@126.com; \\ 'mingnading@126.com; ${ }^{e} 574404587 @ q q . c o m$
}

\begin{abstract}
Database system is mainly created for superhard cutting. In the paper, the processing time is considered as the objective function based on the issue needs, the estcblishing of the mathematical model of the cutting database system mainly takes cutting force power tools, precision machining and tool life as constraints. And the database develops through the development software VS2008 and SQL Server 2008 datobase management system, using an interactive particle swarm optimization to optimize machine tool and cutting parameters, basing on fuzzy matter-element analysis method establishes the evaluation module of tool cutting of the PCBN tool turning hardened steel, and the papergives evaluation methods.
\end{abstract}

Keywords: Superhard Cutting, Cutting Database, Interactive Particle Swarm, Fuzzy Matter Element

\section{Introduction}

Cutting database is the product of the combination that contemporary computer technology of rapid development and raditional machining technology, it has accumulated and stored a wealth production and test data of metal cutting, and it manages and maintains these datas by computer; according to the theoretical and empirical models, for the requirements of users, it can quickly process and draw too parameters and cutting parameters [1]. Currently, to meet a wide variety of processing methods appearing the producing scene and the workpiece material and tool material or properties changing in the actual process, the study of metalcutting machining database has gradually developed from traditional development obtaining static cutting parameter data into dynamic database based artificial intelligence[2].

$\mathrm{CBN}$ as a modern superhard material, as has many excellent properties, has been widely used in the machining of material of high hardness and difficult to machine shardened steel, high hardness cast iron, tool steel and so on. Nowadays, with the CBN superhard materials constantly stydied, many of the world leading tool manufacturers have launched a series of new PCBN cutting tools, thus the study of PCBN cutting tool has become a hot research [3]. E aluation and analysis of cubic boron nitride $(\mathrm{CBN})$ tools product level, is written by Sichuan University Professor Kou Zili, by selecting the American GE PCBN tools and China PCBN tools and using the Delphi method to evaluate the quality level of the tool, comes to a conclusion that PCBN cutting tools come with GE's 20-year gap at the time. This shows that the overall performance and quality of our own production PCBN tools has a large gap with foreign countries, but poor stability.

Superhard cutting database created in this article achieves the optimization of cutting parameters based on interactive particle swarm, and achieves the cutting evaluation of PCBN tools when turning hardened steel by the fuzzy matter element analysis method. 


\section{Overall Design of Superhard Cutting Database}

To make the system more modular and more concise, and reduce system development time, the paper uses object-oriented programming, VS2008 as the programming environment, $\mathrm{C}$ \# as the programming language, SQL Server 2008 as the data management software, and ODBC as data sources to establish a superhard cutting database system. The system mainly includes six modules, as shown in Figure 1. The data query module mainly provides the query of all the cutting tools' data, the data update module can update and improve the database information, the experiment management module can query the experimental program and experimental data, the tool evaluation module and the data optimization module are the core of database, which complete optimization of cutting parameters and evaluation of tool performance, finally, the system maintenance module can protect the security and stability of the system.

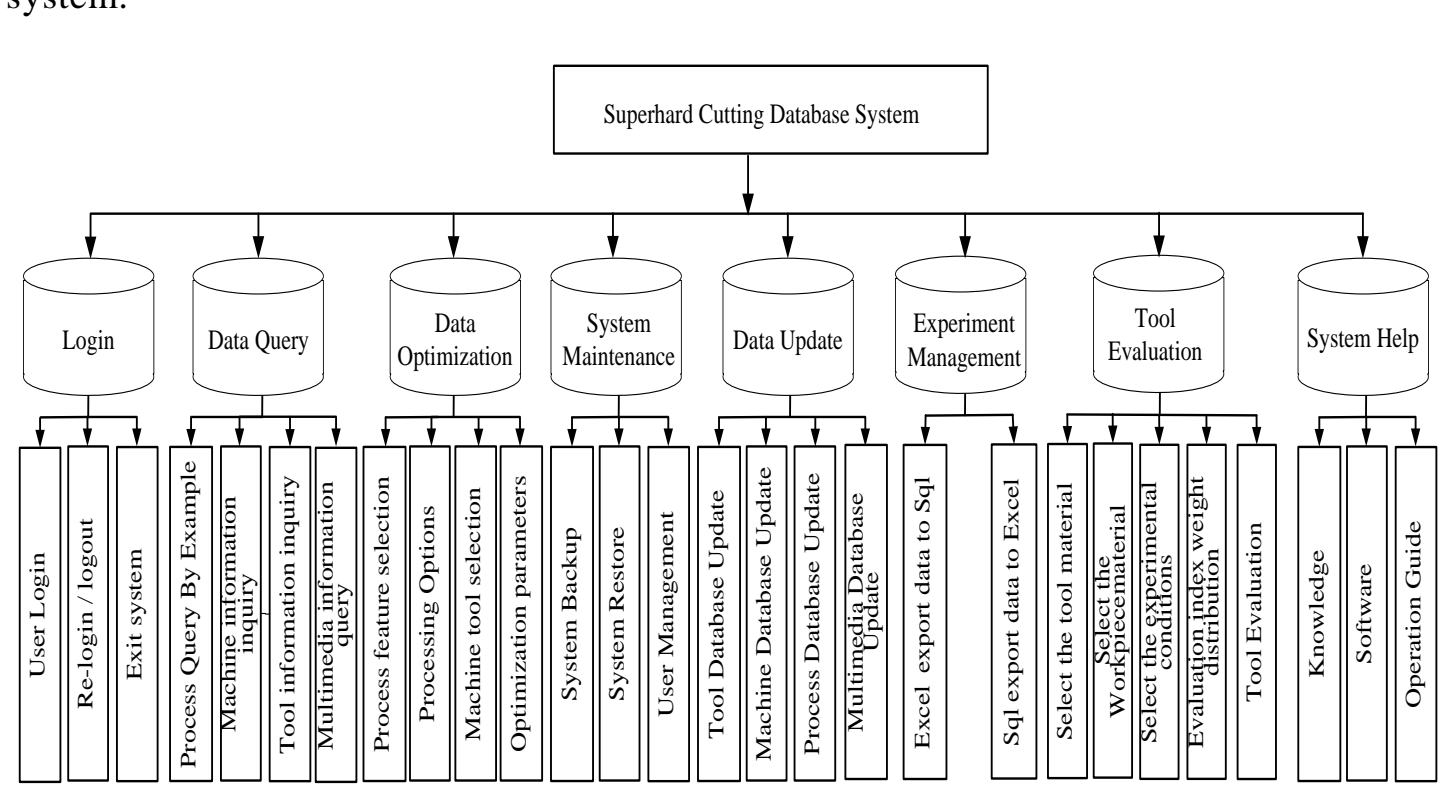

Figure 1. Functional Modules of the System

\section{Establishing of Cutting Preferred Model}

Establishing of cutting preferred model mainly includes determining the optimization goals of cutting amount, establishing the mathematical model and considering constraints. Criteria and indicators preferred cutting parameters is the objective function. Parameter optimization objective function is divided into the lowest production costs, objective function and maximum productivity. In this paper, the highest productivity is taken as a parameter optimization objective function.

The mathematical model of processing time with the highest productivity as parameter optimization objectives is:

$$
t=t_{m}+\frac{t_{c t}}{N_{p}}+t_{0}+t_{n}+t_{i}+\frac{t_{d}}{N_{p}}
$$

Where: $t$-processing time(min); $t_{c t}$ a tool change time(min); $t_{m}$-cutting time(min); $t_{n}$-rapid moving time(min); $t_{0}$-process auxiliary time $(\min ) ; t_{d}$-adjustment time(min); $t_{t}$-tool indexing time(min); $N_{p}$-number of processing workpiece in a tool change time. 
1) For turning cutting time $t_{m}$

$$
\begin{gathered}
t_{m}=\frac{L+e}{n f}=\frac{(L+e) \pi d}{1000 v f} \\
n=\frac{1000 v}{\pi d}
\end{gathered}
$$

Where: $e$-empty travel distance $(\mathrm{mm}) ; n$-speed $(\mathrm{r} / \mathrm{mm}) ; v$-cutting speed $(\mathrm{m} / \mathrm{min}) ; L$-travel distance of tooling a product $(\mathrm{mm})$.

2) Tool change time $t_{c t}(\min )$ :

a. Tool regrinding, $t_{c t}=$ Tool handling time + Brothers Time

b. Machine folder indexable throwaway knives,

$$
t_{c t}=\frac{\text { Tool indexing time } \times(\text { Each average availability Flutes }-1)}{\text { Each average availability Flutes }}
$$

3) Rapid traverse time $t_{n}(\min )$ :

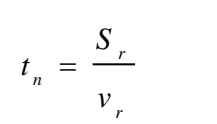

Where: $v_{r}$-Feed mechanism quickly moving speed $(\mathrm{mm} / \mathrm{min}) ; S_{\text {, }}$-Fast-moving total distance(mm).

4) Number of processing workpiece in a tool change time, the formula is expressed as:

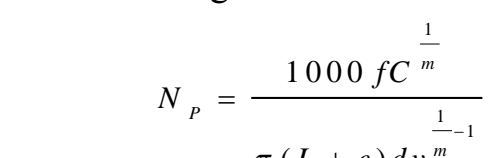

$T$ - tool life(min), the formula is expressed as.

$$
v T^{m}=C
$$

Where: $m$ - index, indicates the influence degree of the $v$ - $T$; $C$ - modulus, is related with the tool, workpiece material and cutting conditions.

The Eq. (2), (3), (4), (5), (6) ane taken into Eq. 1 to obtain the objective function of turning, as shown in Eq. ( 7 :

$$
\frac{(L+e) \pi d}{1000 f v}+\left(t_{c t}+t_{d}\right) \frac{(L+e) \pi d v^{\left(\frac{1}{m}\right)-1}}{1000 f C^{\frac{1}{m}}}+t_{0}+t_{n}+t_{i}
$$

Then Eq. (7) is $\frac{\partial t}{\partial v}=0$, in order to obtain the ${ }^{v_{0}}$ which maximum productivity is corresponding to:

$$
v_{0}=\frac{C}{\left\{\left\lceil\left(\frac{1}{m}\right)-1\right]\left(t_{c t}+t_{d}\right)\right\}^{m}}
$$

In this case, the shortest processing time $t_{0}$ is:

$$
t_{0}=\frac{(L+e) \pi d}{1000 f C}\left(\frac{t_{c t}+t_{d}}{m}\right)^{m}(1-m)^{m-1}
$$


The system constraints considered are cutting force, precision, power tools and tool life, in which the tool life is as a test condition.

\section{Establishing of Cutting Optimization Model Based Optimization}

In the system, a preferred method of interactive is used to select machine and tool, shown in Figure 2. PSO is used to achieve the optimization of cutting parameters.

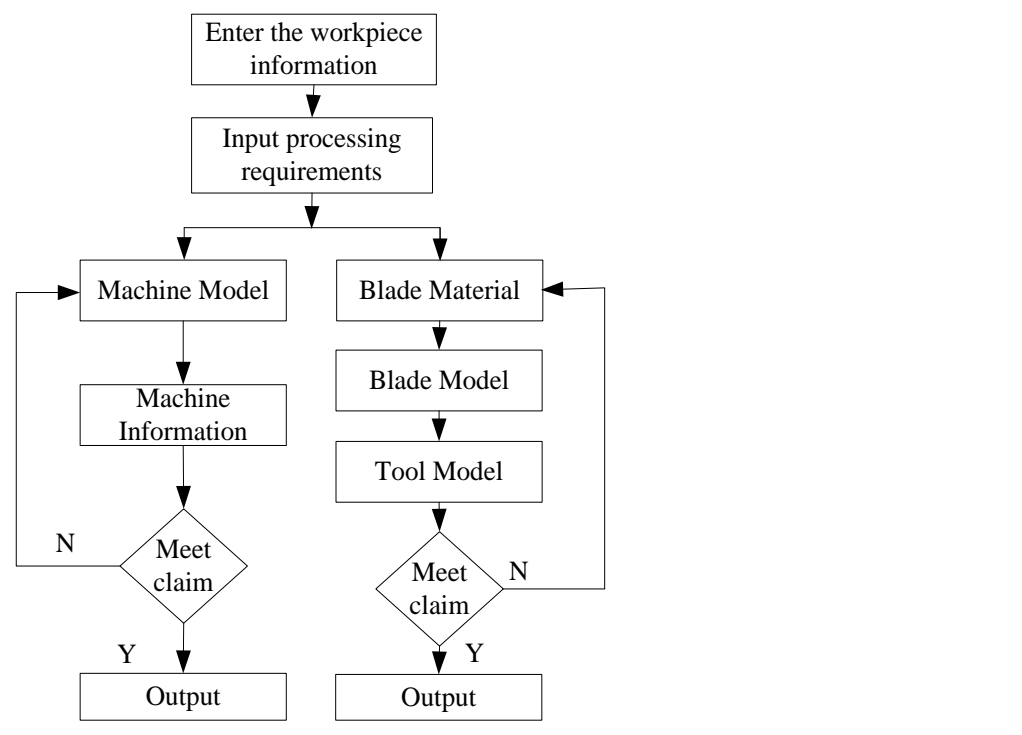

Figure 2. Flowchart of hiteractivePreferred of Machine and Tool

PSO (Particle Swarm Optimization, PSO) algorithm was first jointly proposed in 1995 by the American social psychoogist James Kennedy and electrical engineer Russell Eberhart. The basic idea of the algorithm is: in a d-dimensional space, assuming that there is a community of $\mathrm{m}$ particles, each particle has three attributes: location $X_{i}=\left(x_{i 1}, x_{i 2} \cdots \cdots x_{i d}\right)$, speed $V_{i}=\left(v_{1, n_{i}} \cdot \cdots v_{i d}\right)$ and the fitness value.

The position of the particle is one potential solution of the optimization problem, the velocity characterize the flight direction and distance of current particle, the fitness value is used to measure the supe ior degree of particle's current position. After each iteration of this community, three properties of particles will be an update to certain rules, namely, by tracking individual extreme pBest and global extreme gBest to update themselves [4]. The basic formula of PSO algorithm is following:

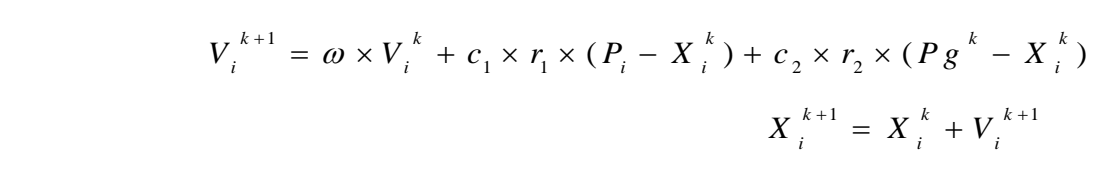

PSO principle is shown in Figure 3. 


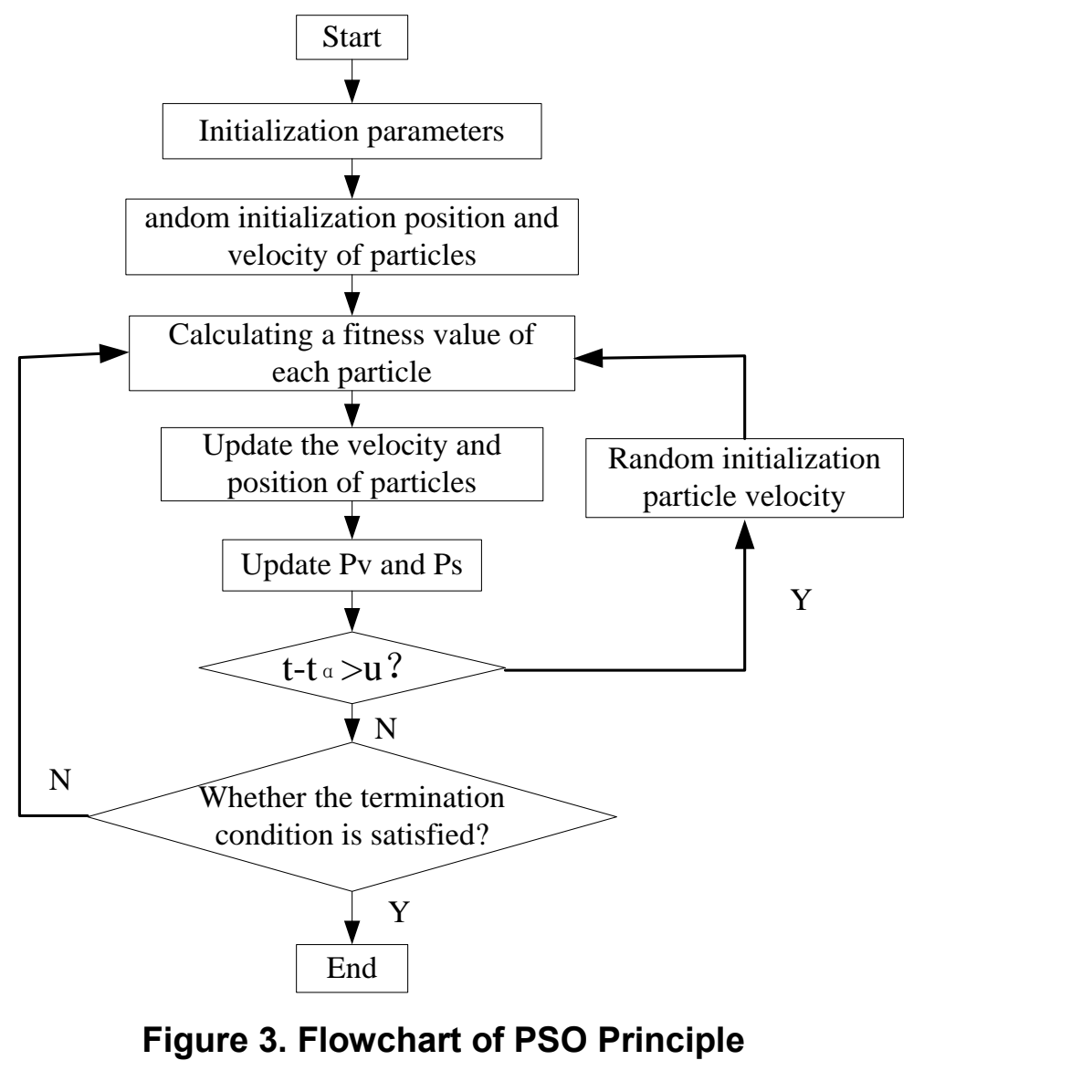

\section{Research on Tool Performairce Evaluation Model of PCBN Turning Hardened Steel}

PCBN cutting tool is artificia CBN tool, at high temperature, it can still maintain the characteristics of high hardness, and is mainly used to do with the processing of iron. PCBN with its superior cutting performance is applied to various fields, especially in the processing of high hardness materials, cutting difficult materials is unique. After development and application of 30 years now PCBN tool material has emerged for the processing of different materials.

In this paper, modern cutting theory, mathematical modeling and model analysis that fuzzy matter-element method are used to establish the tool performance evaluation model of PCBN turning hardened steel for different workpiece materials, application conditions and processing requirements. Tool performance evaluation of PCBN turning hardened steel mainly evaluates tool life, processing quality, processing efficiency, economy and other aspects of processing.

Points of matter element analysis are that things are described with "things, features, value" these three elements, in order to do qualitative analysis and quantitative calculations for things. If its magnitude is fuzzy, they will form a "thing, features, fuzzy values" ordered triples, and this matter element is called fuzzy matter element [5], which is denoted: 


$$
\underline{R}=\left(\begin{array}{cc} 
& M \\
C & \mu(x)
\end{array}\right)
$$

Where: $M$ is things, $C$ is characteristics of things, $\mu(x)$ is fuzzy magnitude that is corresponding with characteristic $C$, scilicet the membership of $x$ that things $M$ is corresponding to its characteristic $C$ values.

When evaluate PCBN cutting tool, if the "PCBN tool cutting" as "things", the value "cutting evaluation" as things "features", the value of evaluation as "value", it will constitute ordered triple on PCBN cutting tool.

1) Determining method of cutting performance evaluation membership

The membership of each index is basis constructed evaluation model of PCBN cutting tool fuzzy matter-element. In order to comprehensive evaluation of multiple targets, we need to take membership of each index as the standard of measuring PCBN cutting tool. In order to establish an optimized comparison standard, we need develop an optimization principle, which measures by merit membership of individual feature as a standard. The so-called merit membership is fuzzy values corresponding to characteristic of each indi idual belonging to the fuzzy values of features that standard things are corresponding to.

In the fuzzy matter-element analysis, if "magnitude" of characteristics is a specific value, you do not need to introduce the membership function. Finally, the evaluation of PCBN tool cutting is based on the evaluation of each specific numerical to describe. When calculating each evaluation index membership, just dealing with "Value" according to the principles of merit and the "values" change into mentbership, we will get membership values various evaluation corresponding to.

2) Evaluation index weight analysis

Weight is the amount which reflects the affecting degree that each index treats evaluation things, the weight is di ided into subjective weight and objective weight. Subjective weight analys and determines the impacting extent of each evaluation treating things by the understanding of evaluator treating things, after weighing, assigning the weight to the evaluation. The objective weight is obtained by using statistical methods to calculate based on inherent information of values of each index. In order to fully reflect the importance of the evaluation index of PCBN tool cutting, this paper determine the each index weight by combining subjective weight and objective weight.

Assuming each index subjective weight is $\theta_{j}(j=1,2, \cdots \cdots, n)$, so

$$
\mathcal{N} \sum_{j=1}^{n} \theta_{j}=1
$$

Betermining the method of the objective weight is a lot, this paper uses the summation nornalization method. First, calculating the arithmetic average of the correlation coefficients, and then calculating the sum of arithmetic average of the correlation coefficients of evaluations, and finally using the the normalization process for arithmetic average of the correlation coefficient of evaluations. The objective weight of each evaluation is: 


$$
W_{j}^{\prime}=\frac{\sum_{j=1}^{n} \xi_{j i}}{\sum_{j=1}^{n} \sum_{i=1}^{m} \xi_{j i}}
$$

Integrating Eq.11,12, the final weight of each evaluation is:

$$
W_{j}=\frac{\theta_{j} W_{j}^{\prime}}{\sum_{j=1}^{n} \theta_{j} W_{j}^{\prime}}
$$

Where

$$
\sum_{j=1}^{n} W_{j}=1
$$



$W_{j}$ is main and objective portfolio weight.

\section{Summary}

This paper establishes a superhard cutting database based on cutting parameters optimization and the evaluation of PCBN too cutting aiming to superhard cutting. The system can obtain reasonably cutting parameters throughinteractive particle swarm and use fuzzy matter-element analysis method to study the evaluation model of tool cutting when PCBN tool turns hardened steel. The system has many advantages, as rational design, functional, simple and beautiful system interface, easy and flexible operation, low technical requirements for operating personnel. This database system can provide a reasonable or optimized cutting data for superhard cutting and have a scientific and accurate evaluation for PCBN tool cutting. System has great significance for improving productivity and processing of enterprises.

\section{Acknowledgements}

The work presented in this paper was supported by the National Natural Science Foundation of China (Grant No.51105118); the Program for New Century Excellent Talents in University (GranTNo.NCET-10-0146) and the Science and Technology Research Projects of Education Department of Heilongjiang province (Grant No.12521077).

\section{Reference}

[1] L. X. Liu, W. J. Zheng, M. Liu, Z. Chen and H. P. Hui, "J. Optimization and Application of Cutting Database", Journal of Harbin University of Science and technology, vol. 1, no. 18, (2013).

[2] M. Liu, M. Ding, X. Liu, Z. Chen and Y. Wang, "Key Engineering Materials", Design and Evaluation Function Block of Inserts for Heavy Cutting, (2014), pp. 168-172; Xiamen, Fujian .

[3] Y. W. Dou, Z. L. Kou, L. Qi and D. W. He, “J. Turning Hardened Bearing Steel with PCBN Tool”, Diamond \& Abrasives Engineering, vol.6, no. 30, (2010).

[4] Q. Zhang, Z. T. Chen, P. Zhang and L. Wang, "J. Cutting Parameters Based on Particle Swarm Optimization and Constrained Process", Aviation Precision Manufacturing Technology, vol. 1, no. 46, (2010).

[5] H. Z. Ma, "High Speed Milling Cutter Cutting Performance Evaluation Based on Fuzzy-Matter Element", Harbin University of Science and Technology, (2008). 
International Journal of Hybrid Information Technology

Vol.7, No.5 (2014)

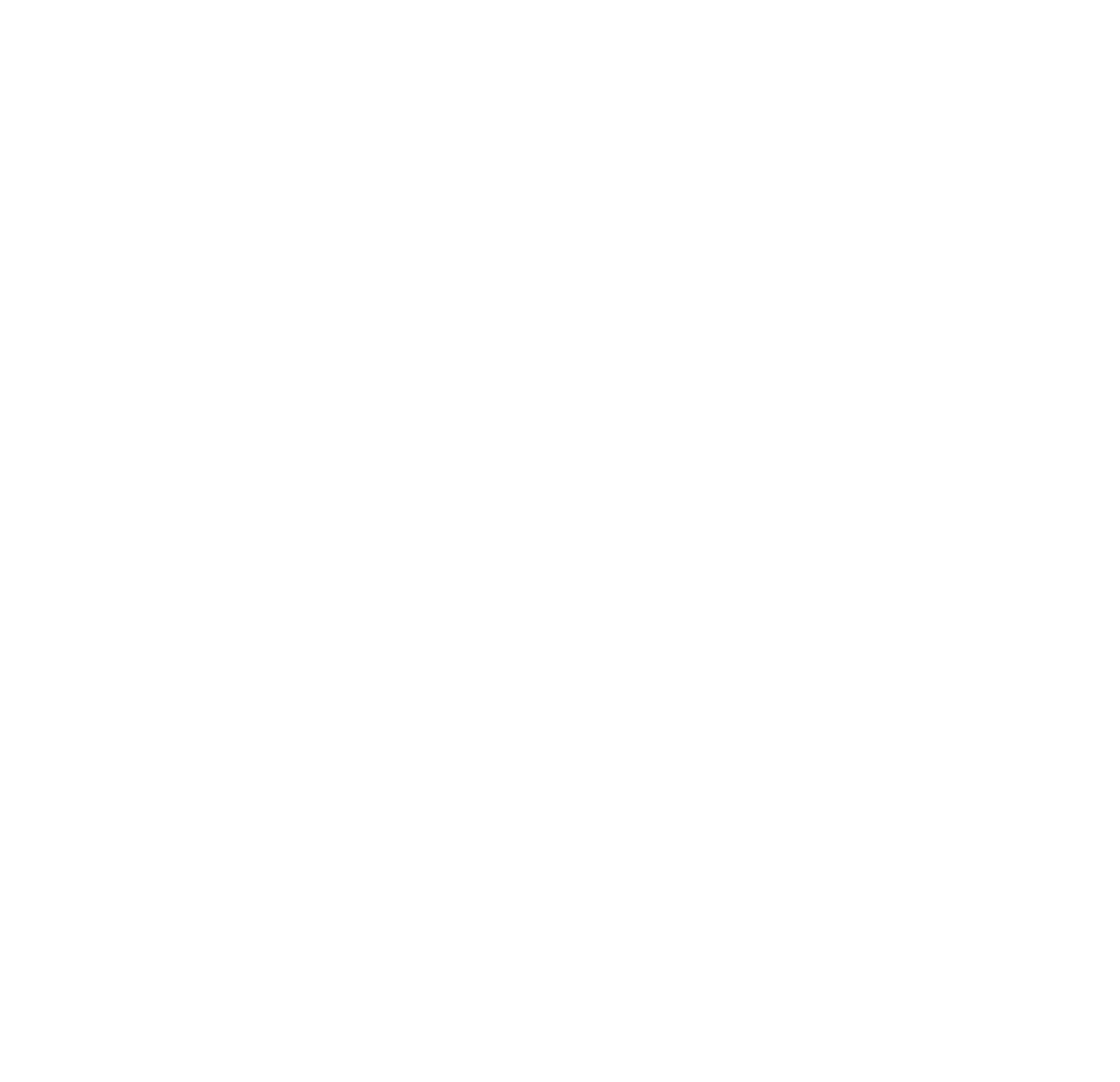

